\title{
Wave turbulence on the surface of liquid hydrogen in restricted geometry: the influence of the boundary conditions
}

\author{
M.Yu. Brazhnikov, A.A. Levchenko, L.P. Mezhov-Deglin, and I.A. Remizov \\ Institute of Solid State Physics RAS, Chernogolovka, Moscow District 142432, Russia \\ E-mail: levch@issp.ac.ru
}

Received February 24, 2015, published online April 23, 2015

\begin{abstract}
Formation of low frequency harmonics on turbulent distribution in the system of waves on the surface of liquid hydrogen has been studied in the frequency range 1-100 Hz (capillary-gravity waves). It is shown that the geometry of the experimental cell has a significant influence on the direct cascade of capillary waves generated by monochromatic force as well as on the direction of the wave energy transfer from the range of pumping towards that of dissipation. Besides a direct turbulent cascade, single half-frequency harmonic generation was observed in a cylindrical cell under high pump power. In a square cell we observed not only a half-frequency harmonic but a number of low frequency harmonics below the driving frequency generated by the nonlinear threewave interaction. In the case of a rectangular cell we observed formation of incommensurate low frequency harmonics caused by the three-wave interaction of capillary waves and generation of a wave mode of $\sim 1 \mathrm{~Hz}$ in the frequency range of gravity waves which could be attributed to the four-wave interaction.
\end{abstract}

PACS: 61.30.-v Liquid crystals;

47.27.Gs Isotropic turbulence; homogeneous turbulence.

Keywords: liquid hydrogen, wave turbulence, low frequency harmonics.

\section{Introduction}

The dispersion law for waves on a free surface of liquid in unrestricted geometry is given by the expression

$$
\omega^{2}(k)=g k+\sigma / \rho k^{3}
$$

where $\omega$ and $k$ are the frequency and the wave vector length of the surface wave, $\sigma$ and $\rho$ are the surface tension and density of liquid, $g$ is the free-fall acceleration. The first term in expression (1) describes pure gravity waves, the second term corresponds to capillary waves. For capillary waves the main processes of their nonlinear interaction are the three-wave processes of decay and merging, and for gravity waves the main process is the four-wave interaction [1]. All the processes should satisfy the energy (frequency) and the wave vector conservation laws.

In an experimental cell of finite size the spectrum of surface waves becomes discrete. The distance between the two nearest resonances in $k$-space is determined by the boundary conditions and in the general case it is inversely proportional to cell size $D$. In $\omega$ space the distance between two resonances $\Delta \Omega$ for capillary waves increases as $\Delta \Omega \sim \omega^{1 / 3}$ and decreases for gravity waves, $\Delta \Omega \sim \omega^{-1}$. Due to the discreteness of the eigenmodes of the surface oscillations of liquid in a basin of finite size the frequency and wave vector conservation laws cannot always be satisfied, for instance for capillary waves in a rectangular basin three-waves processes are prohibited [2]. In real systems strict restrictions are softened due to viscous $\omega_{v}$ and nonlinear $\omega_{n}$ broadening of the resonances [3,4], and the conservation laws for the three-wave interaction could be rewritten in the form

$$
\begin{gathered}
\omega_{1}+\omega_{2}=\omega_{3}+\delta \\
\boldsymbol{\kappa}_{1}+\boldsymbol{\kappa}_{2}=\boldsymbol{\kappa}_{3}
\end{gathered}
$$

where $\Delta \Omega>\Delta \omega=\Delta \omega_{v}+\Delta \omega_{n}$, and $|\delta|<\Delta \omega$.

In the case of radial waves on the surface of cylindrical cells we deal with one-dimensional space, the wave vector $\boldsymbol{\kappa}$ might be considered as an integer and the spectrum of capillary waves can be described by the expression

$$
\omega=(\sigma / \rho)^{1 / 2}(2 \pi / D)^{3 / 2}(n-3 / 4)^{3 / 2}
$$

where $n$ is the resonance number. 
When using square or rectangular cells the $\boldsymbol{\kappa}$ space becomes two-dimensional. The resonant frequencies for the capillary waves in the rectangular cell are described by the expression:

$$
\omega=(\sigma / \rho)^{1 / 2}\left[(\pi n / a)^{2}+(\pi m / b)^{2}\right]^{3 / 4}
$$

and for the standing gravity waves:

$$
\omega=g^{1 / 2}\left[(\pi n / a)^{2}+(\pi m / b)^{2}\right]^{1 / 4}
$$

where $a$ and $b$ are the length and width of the working cell, $n$ and $m$ are the integer numbers.

In our experiments the excitation of surface waves was performed in the frequency range from 10 to $100 \mathrm{~Hz}$. It should be noted that waves with frequencies from 1 to 100 $\mathrm{Hz}$ on the surface of liquid hydrogen should be considered as capillary-gravity ones and the three-wave processes along with the four-wave ones are not prohibited for them at frequencies lower or higher some critical frequency $f_{C}$

$$
f_{0}=\frac{1}{2^{1 / 2} \pi}\left(\frac{g^{3} \rho}{\sigma}\right)^{1 / 4}
$$

It is clear that the capillary waves correspond to frequencies $f>>f_{C}$, and at $f<<f_{C}$ we deal with the gravity waves on the liquid surface. For liquid hydrogen $f_{C}=16 \mathrm{~Hz}$ at $T=15.6 \mathrm{~K}$.

In the turbulent state the energy pumped into the system by an external source is transferred from the pumping region to the domain of dissipation owing to the nonlinear wave interaction. The theory [1] predicted that in a system of capillary waves in unrestricted geometry only direct turbulent cascade should be generated and the wave energy should flow towards the high frequency domain. In a system of gravity waves formation of the both direct and inverse cascades is possible and the wave energy could be directed into both high and low frequency domains. From our experiment and subsequent numerical simulations [5-7] it follows that in a finite size cell, where the spectrum of the wave excitations is discrete, one might observe an energy flow from the pumping region towards both high and low frequency domains even for capillary waves. It should be mentioned that in all the numerical simulations the authors preferred to use asymptotic approximations, $\omega \sim \kappa^{1 / 2}$, for capillary waves and $\omega \sim \kappa^{3 / 2}$ for gravity waves. For the case of our experimental conditions when the capillary-gravity waves prevail in the cells, especially in the range of $120 \mathrm{~Hz}$, the dispersion law of wave excitations on the charged hydrogen surface is close to linear $\omega \sim \kappa$ [8], numerical simulations being absent.

In this paper we present the recent results of our investigations of the wave turbulence on the charged surface of liquid hydrogen in the cells of three different geometries, namely, cylindrical, square and rectangular cells of the same depth and comparable dimensions. The main goal of this study was to clarify the influence of the boundary conditions on the turbulent spectra and mechanisms of wave energy transfer in the system of surface waves in restricted geometry.

\section{Experimental technique}

The experiments were carried out at constant temperature $T=15 \mathrm{~K}$. Hydrogen gas was condensed into a copper cup of inner diameter $60 \mathrm{~mm}$ and depth $4 \mathrm{~mm}$. Square and rectangular cells were formed by flat metal inserts into the original cylindrical cell. The inner dimensions of the square cell were $40 \times 40 \mathrm{~mm}$, and those of the rectangular one $40 \times 20 \mathrm{~mm}$. The metal collector (copper plate) suspended at a distance of $4 \mathrm{~mm}$ above the experimental cell and surface of liquid hydrogen formed a flat capacitor. A source of charges was placed at the bottom of the cup. The liquid hydrogen surface was charged with positive ions when dc voltage of positive polarity $U_{d c} \approx 1 \mathrm{kV}$ was applied between the copper cup and collector. Lowfrequency ac voltage $U_{a c}=U_{p} \sin (\omega \mathrm{t})$ applied to the cup in addition to the dc voltage excited waves on the charged surface of liquid hydrogen. It should be noted that waves on the charged surface are generated owing to a strong spatial heterogeneity of the electric field near the edges of the experimental cell.

The surface waves were detected by means of a laser beam reflected from the liquid surface and then focused onto a photodetector. Detailed method of registration of surface elevations is given in [5]. Any variations of the angle between the gliding laser beam and the oscillating surface led to modulation of the power of the reflected beam. The ac signal from the photodetector was amplified and digitized by a 24-bit analog-to-digital converter with sampling frequency $102.4 \mathrm{kHz}$. The amplitude of digitized signal $P(t)$ is proportional to power variation of the reflected laser beam. As it was shown earlier [9], power spectrum $P_{\omega}^{2}$ is proportional to the spectrum of the pair correlation function of the surface elevation $\left\langle\eta_{\omega}^{2}\right\rangle$. The angular amplitudes of the waves in our experiments were close to the maximum allowed value in the geometry of our experiment which is equal $0.05 \mathrm{rad}$.

It is necessary to note that the applied perpendicular electric field was less the critical value at which the flat charged surface of liquid loses its stability. Moreover, the perpendicular electric field modifies the dispersion law of the surface waves: in our experiments the fundamental frequency is shifted to low frequencies compared to Eq. (1).

\section{Results and discussion}

\section{Cylindrical cell}

The power spectra of oscillations $P_{\omega}^{2}$ on the surface of liquid hydrogen in the cylindrical cup presented in Figs. 1 and 2 show changes in the turbulent distribution $0.3 \mathrm{~s}$ and 


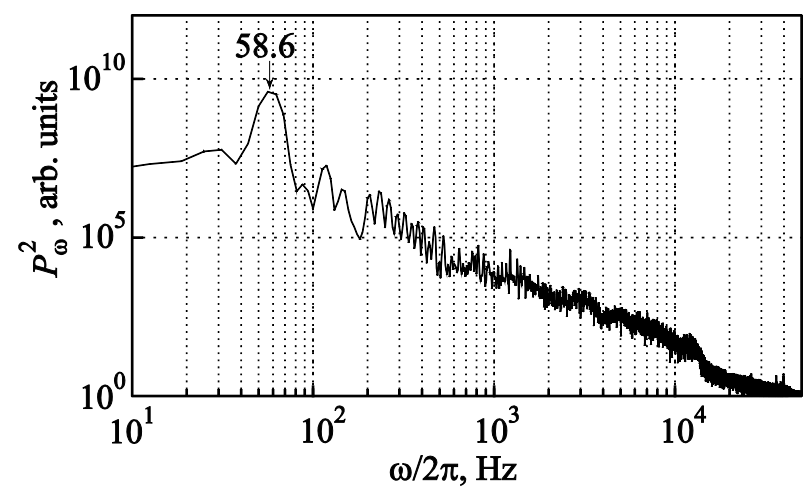

Fig. 1. Turbulent cascade on the surface of liquid hydrogen in the cylindrical cup $0.45 \mathrm{~s}$ after switching on pumping at frequency $58.6 \mathrm{~Hz}$.

32 s after switching on monochromatic driving force (pump) at the frequency of the fifteenth resonance: $f_{p}=\omega_{p} / 2 \pi=$ $=58.6 \mathrm{~Hz}$. The power spectrum $P_{\omega}^{2}$ was obtained by processing the $P(t)$ dependence recorded over a time window of duration $\Delta t=0.3 \mathrm{~s}$. In Fig. 1 the main peak at driving frequency $\omega_{p}$ dominates in distribution $P_{\omega}^{2}$, and the direct turbulent cascade extends up to $10 \mathrm{kHz}$. At frequencies above $2 \mathrm{kHz}$ distribution $P_{\omega}^{2}$ displays power-law dependence $P_{\omega}^{2} \sim \omega^{2}$ with exponent $n$ close to -2.5 . This value of exponent $n$ is closer to the value $n$ predicted by the theory [1] for the case of broadband pumping. At frequencies close to and above $10 \mathrm{kHz}$ we observed a high frequency edge of the inertial interval.

Within $32 \mathrm{~s}$ the turbulent cascade shown changed significantly. In Fig. 3 one can see the appearance of a low frequency subharmonic at half driving frequency $\omega_{0} / 2$ and additional combination harmonics like $\omega_{p}+\omega_{p} / 2$, etc. in the direct cascade. The three peaks at frequencies $\omega_{p} / 2, \omega_{p}$ and $\omega_{p}+\omega_{p} / 2$, which dominate in the low frequency domain play the role of a wide pumping range, although the surface is excited by an electric force at single frequency $\omega_{p}$. The turbulent distribution over the frequency range from $100 \mathrm{~Hz}$ to $10 \mathrm{kHz}$ can be described by a power law

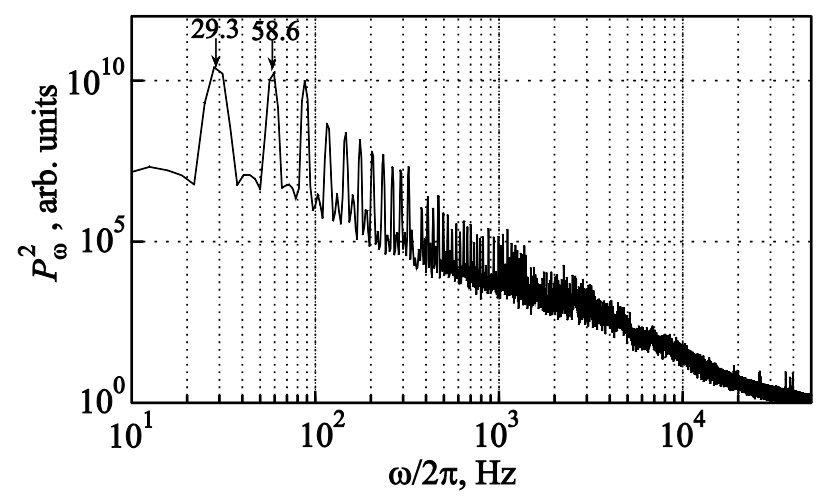

Fig. 2. Turbulent cascade on the surface of liquid hydrogen in the cylindrical cup $32 \mathrm{~s}$ after switching on pumping at frequency $f_{0}=58.6 \mathrm{~Hz}$.

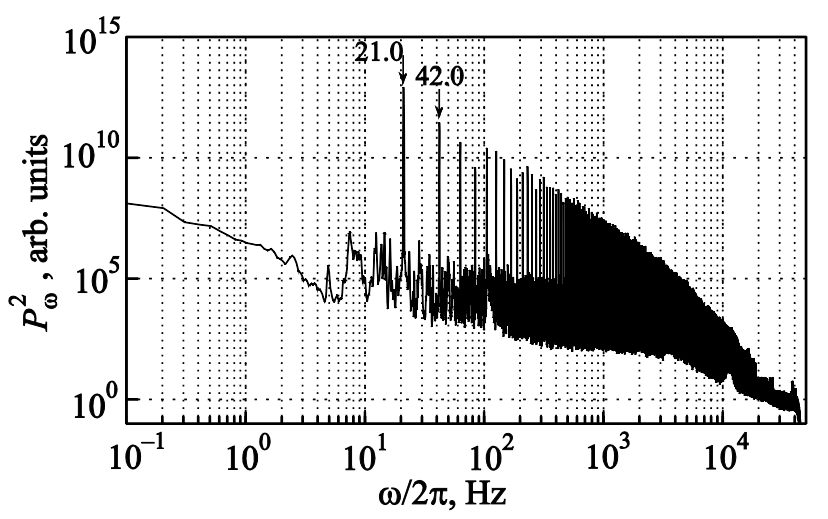

Fig. 3. Turbulent cascade on the surface of liquid hydrogen in the square cell. Excitation frequency $f_{p}=\omega_{0} / 2 \pi=42 \mathrm{~Hz}$.

function with an exponent close to - 3. It is seen that the dissipative domain of the turbulent distribution can be well described by an exponential dependence of the frequency with the characteristic frequency of about $10 \mathrm{kHz}$, close to the high frequency edge of the inertial range $[10,11]$. The time of appearance of the subharmonic at half-frequency harmonic $f_{s}=f_{0} / 2$ in distribution $P_{\omega}^{2}$ depends on the amplitude of the driving force and varies from several seconds to several minutes after switching on the excitation.

\section{Square cell}

Figure 3 shows turbulent distribution $P_{\omega}^{2}$ in the system of waves on the surface of liquid hydrogen excited by harmonic force at frequency $42 \mathrm{~Hz}$ marked by the arrow. At low frequencies there is a half-frequency peak at frequency $\omega_{0} / 2=21 \mathrm{~Hz}$ with an amplitude much higher than that at the frequency of excitation $\omega_{0}$. The frequency dependence of the turbulent spectrum is very close to $\omega^{-3}$ within the frequency range from $100 \mathrm{~Hz}$ to $2 \mathrm{kHz}$. The dissipation interval is observed at frequencies higher than $2 \mathrm{kHz}$.

When the frequency of excitation was decreased to $41.8 \mathrm{~Hz}$ (Fig. 4) at the same excitation amplitude dramatic events occurred in the turbulent distribution. In addition to the half-frequency harmonic we observed two other peaks at high amplitude frequencies of 30.6, 20.9, 11.4, 9.7. In the low frequency domain there are two high amplitude peaks at frequencies $1.7 \mathrm{~Hz}$ and $3.5 \mathrm{~Hz}$. Near the peaks we can also see numerous satellite harmonics. We suggest that the wave at frequency $f_{p}=\omega_{p} / 2 \pi=41.8 \mathrm{~Hz}$ decays into two peaks with frequencies $11.4 \mathrm{~Hz}$ and $30.6 \mathrm{~Hz}$. And the wave at frequency $11.4 \mathrm{~Hz}$ decays into two waves with frequencies $1.7 \mathrm{~Hz}$ and $9.7 \mathrm{~Hz}$. The remaining low frequency harmonics are the result of the nonlinear wave interaction:

$$
\begin{gathered}
41.8 \mathrm{~Hz} \Rightarrow 11.4 \mathrm{~Hz}+30.6 \mathrm{~Hz} \\
11.4 \mathrm{~Hz} \Rightarrow 1.7 \mathrm{~Hz}+9.7 \mathrm{~Hz}
\end{gathered}
$$




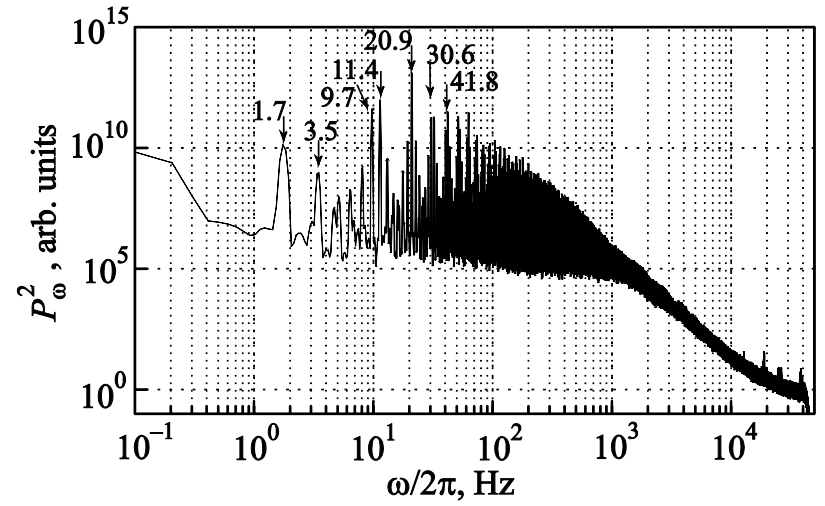

Fig. 4. Turbulent cascade on the surface of liquid hydrogen in the square cell. Excitation at frequency $f_{p}=\omega_{0} / 2 \pi=41.8 \mathrm{~Hz}$.

\section{Rectangular cell}

In the rectangular cell a developed turbulent cascade is easily excited well under pumping in the frequency range 10-40 Hz. In Fig. 5 we present distribution $P_{\omega}^{2}$ when the surface of the liquid in the rectangular cell was excited by a harmonic force at frequency $f_{p}=\omega_{p} / 2 \pi=28.0 \mathrm{~Hz}$, as indicated by the arrow. The half-frequency peak at frequency $f_{p} / 2=14 \mathrm{~Hz}$ is well seen. Note that its amplitude is much higher than that of the peak at the pumping frequency. The origin of this harmonic is the decay of the main peak into two,

$$
28 \mathrm{~Hz} \Rightarrow 14 \mathrm{~Hz}+14 \mathrm{~Hz} \text {. }
$$

As the frequency of pumping is increased by $0.5 \mathrm{~Hz}$, the turbulent cascade is significantly modified (Fig. 6). We should point out several observations. First, the main peak decays into two incommensurate harmonics:

$$
28.5 \mathrm{~Hz} \Rightarrow 9.9 \mathrm{~Hz}+18.7 \mathrm{~Hz} \text {. }
$$

Second, in the low frequency range there is a peak with a frequency equal to $1.1 \mathrm{~Hz}$. The wave length of this mode is exactly equal to the first resonant mode of the surface oscillations along the long side of the experimental cell. This peak should be attributed to the four-wave interaction like

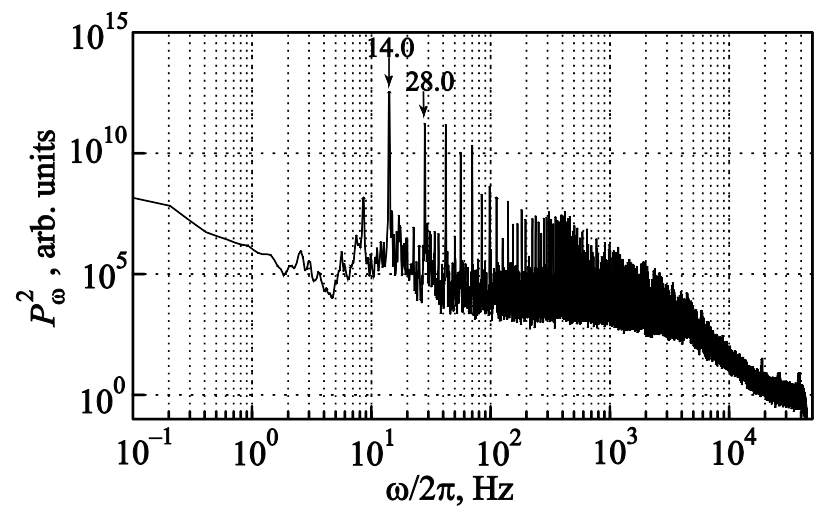

Fig. 5. Turbulent cascade on the surface of liquid hydrogen in the rectangular cell. Excitation at frequency $f_{p}=28 \mathrm{~Hz}$.

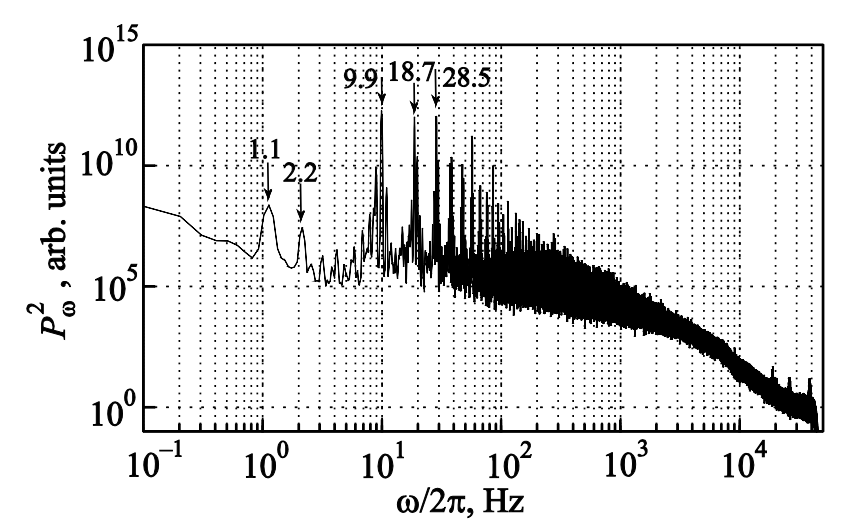

Fig. 6. Turbulent cascade on the surface of liquid hydrogen in the rectangular cell. Excitation at frequency $f_{p}=28.5 \mathrm{~Hz}$.

$$
9.9 \mathrm{~Hz}+9.9 \mathrm{~Hz} \Rightarrow 18.7 \mathrm{~Hz}+1.1 \mathrm{~Hz} .
$$

Third, the lowest subharmonic itself generates a series of other harmonics with frequency multiples of $1.1 \mathrm{~Hz}$. The peaks at frequencies from $9.9 \mathrm{~Hz}$ and higher are surrounded by satellites which result from the interaction of the harmonics with lowest frequency mode $\omega=1.1 \mathrm{~Hz}$. In principle, we can state that we have observed transport of wave energy from the range of excitation to the lowest mode of the experimental cell (resonator) as a result of the four-wave interaction.

In Fig. 7 we show the time dependence of the amplitude of Fourier harmonics in the turbulent cascade observed after stepwise lowering of the frequency of the exciting force from 29.3 to $28.5 \mathrm{~Hz}$. During the first $75 \mathrm{~s}$ the surface was excited by a monochromatic force at frequency $f=29.3 \mathrm{~Hz}$. And at the 75th s the frequency of excitation was lowered stepwise to $28.5 \mathrm{~Hz}$ without changing the amplitude of the excitation.

After changing the frequency we observed the decay of the amplitudes of the harmonics at frequencies $29.3 \mathrm{~Hz}$ and $1.1 \mathrm{~Hz}$ with characteristic time about $1 \mathrm{~s}$. This time is very close to that of viscous relaxation of capillary waves $\tau_{v}$ at frequency $29.3 \mathrm{~Hz}$ [12]. Note that the low frequency mode

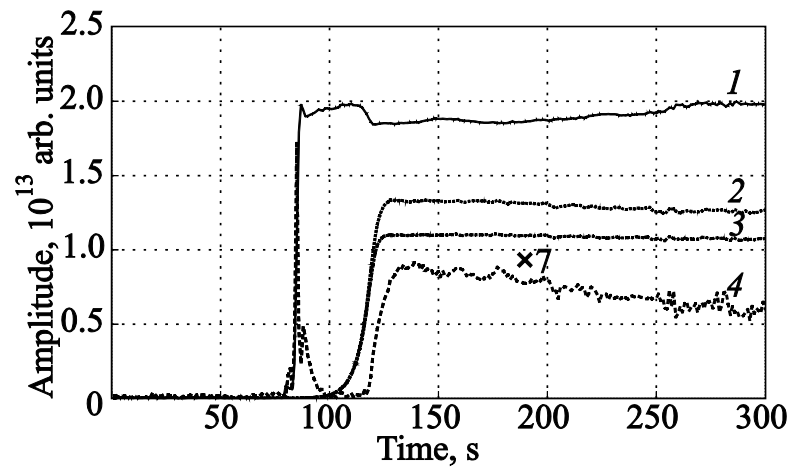

Fig. 7. Time dependence of amplitude of Fourier harmonics generated at the surface of liquid hydrogen in the rectangular cell after changing the frequency of the exciting force from $29.3 \mathrm{~Hz}$ to $f_{p}=28.5 \mathrm{~Hz}: 28.5 \mathrm{~Hz}$ (1), $18.7 \mathrm{~Hz}$ (2), $9.9 \mathrm{~Hz}$ (3), $1.1 \mathrm{~Hz}$ (4). 
was excited exactly at the moment of changing frequency. These peaks disappeared completely by 100 s. However, the low frequency mode appears again at 140th s $65 \mathrm{~s}$ after changing the frequency of the pumping force. Formation of oscillations at frequencies $18.7 \mathrm{~Hz}$ and $9.9 \mathrm{~Hz}$ starts immediately after changing the frequency of the exciting force. It is seen that characteristic time $\tau$ of the three harmonic growth is of the order of several seconds and larger than the time of decay of the main peak. The low frequency peak starts growing when the amplitude of the harmonics at frequencies $9.9 \mathrm{~Hz}$ and $18.7 \mathrm{~Hz}$ reach appreciable values. This experimental fact confirms our assumption that the lowest peak is the result of the four-wave interaction (7).

\section{Conclusion}

From the comparison of results of our measurements obtained at the cells of different geometry it follows that the boundary conditions (shape of the cell) affect significantly the spectra of the wave turbulence on the surface of liquid hydrogen and the direction of the wave energy flow from the range of excitation towards the dissipative domains. At a high excitation level we could observe appearance of a half-frequency subharmonic in the cylindrical cell, while in the case of the square or rectangular cells under similar conditions we observed generation of a number of incommensurate subharmonics down to $\sim 1 \mathrm{~Hz}$ in the low frequency region. The wave energy is transferred from the region of excitation towards low frequencies only by the three-wave interaction in the case of cylindrical and square cells or by the three-wave and four-wave processes in the case of a rectangular cell. Since the waves in the frequency range of $1-20 \mathrm{~Hz}$ correspond to a capillarygravity region, further numerical simulations in this fre- quency range taking into account the real dispersion law for the surface waves in our cells are required.

This work was supported by the Russian Science Foundation, Grant No.14-22-00259.

1. V.E. Zakharov, V.S. L'vov, and G. Falkovich, Kolmogorov Spectra of Turbulence I, Springer-Verlag (1992).

2. Kartashova, Physica D 46, 43 (1990); Kartashova, Physica D 54, 125 (1991).

3. A.N. Pushkarev and V.E. Zakharov, Physica D 135, 98 (2000).

4. V.E. Zakharov, A.O. Korotkevich, A.N. Pushkarev, and A.I. Dyachenko, Pis'ma Zh. Eksp. Teor. Fiz. 82, 544 (2005) [JETP Lett. 82, 487 (2005)].

5. L.V. Abdurahimov, M.Yu. Brazhnikov, A.A. Levchenko, A.M. Lihter, and I.A. Remizov, Fiz. Nizk. Temp. 41, 215 (2015) [Low Temp. Phys. 41, No. 3 (2015)].

6. L.V. Abdurakhimov, M. Arefin, G.V. Kolmakov, A.A. Levchenko, Yu.V. Lvov, and I.A. Remizov, Phys. Rev. E 91, 023021 (2015).

7. A.O. Korotkevich, Pis'ma Zh. Eksp. Teor. Fiz. 97, 145 (2013) [JETP Lett. 97, 126 (2013)].

8. M.Yu. Brazhnikov, L.P. Mezhov-Deglin, A.A. Levchenko, and I.A. Remizov, Pis'ma Zh. Eksp. Teor. Fiz. 100, 754 (2014) [JETP Lett. 100, 669 (2014)].

9. M.Yu. Brazhnikov, A.A. Levchenko, and L.P. MezhovDeglin, Prib. Tekh. Eksp. 6, 31 (2002).

10. L.V. Abdurakhimov, M.Yu. Brazhnikov, A.A. Levchenko, I.A. Remizov, and S.V. Filatov, Pis'ma Zh. Eksp. Teor. Fiz. 95, 751 (2012) [JETP Lett. 95, 670 (2012)].

11. L.V. Abdurakhimov, M.Yu. Brazhnikov, and A.A. Levchenko, Pis'ma Zh. Eksp. Teor. Fiz. 89, 139 (2009) [JETP Lett. 89, 120 (2009)].

12. L.D. Landau and E.M. Lifshitz, Fluid Mechanics. Course of Theoretical Physics, Pergamon Press (1987), Vol. 6. 